\title{
Computational intelligence techniques for reflections identification in iris biometric images
}

\author{
Fabio Scotti \\ University of Milan, Department of Information Technologies \\ via Bramante 65, 26013 Crema (CR), Italy
}

\begin{abstract}
Iris biometric systems identify individuals by comparing the characteristics of the iris acquired by the acquisition sensors. When reflections are present in the iris image, the portion of the image covered by reflections must be discarded from any further comparison since it can produce false matches. The paper presents a methodology for reflections identification in iris biometric images based on neural networks. In particular, this paper proposes a set of features which can be extracted from the iris image and that can be effectively used to achieve an accurate identification of the reflection position using a neural network. Moreover, the paper presents how the radial symmetry operator can be used as a proper feature to identify the reflections in iris images. The method is general and can be used in any biometric system based on iris images.
\end{abstract}

Keywords - Iris, Biometric System, Neural Networks, Radial symmetry.

\section{INTRODUCTION}

Biometric systems exploit automated methods capable to recognize individuals analyzing their physiological and/or behavioral characteristics [1]. Physiological biometrics is based on data derived from direct measurement of a body part (i.e., fingerprints, face, retina, iris), while behavioral biometrics is based on measurements and data extracted from human actions [2] (i.e., gait and signature).

Iris biometric systems perform the identification by processing the following steps. The first step is the acquisition of the iris image (sample) by the sensor module.

The second step is the localization of the iris in the acquired image. All biometric systems can achieve their maximum accuracy in identification or verification [3] only if the samples are correctly acquired. For this reason, all exogenous elements which are superimposed to the real biometric information in the sample must be removed (e.g., in iris images we can have reflections, eyelids and lashes). This operation can reduce the probability of false matching with different individuals. In the case of the iris sample, the exogenous elements are (i) eyelids, (ii) lashes and (iii) reflections. In this step, first of all the edges of the pupils and of the iris must initially be located in the image, then the eyelids, lashes and reflections must be identified and removed from the iris image.

The third step aims at extracting the biometric template (iris encoding) from the iris. This template will be used by the biometric system to perform comparisons (matching) with the others templates used as reference [3], generally stored in a database or in a document.
Typically, the sensor is a CCD-based camera with proper optics. Such systems can achieve a remote acquisition $(60 \mathrm{~cm})$ of a face portion containing one complete eye (Figure 1). Most of the systems require about 100 pixel of resolution for the iris in order to suitably identify a person. Nowadays, lowcost sensors, such as webcams, have been also considered. Figure 1 shows different iris images. Image (a) and (b) have been acquired with cameras working in the IR band (using a macro and a tele-optic, respectively), image (c) has been produced by a low-cost sensor, image (d) and (f) show two irises acquired using visible light (ambient light and lamp light, respectively), and image (e) using a color webcam.

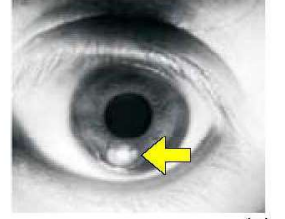

(a)

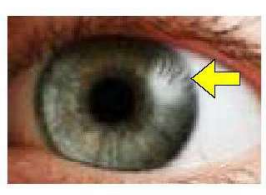

(d)

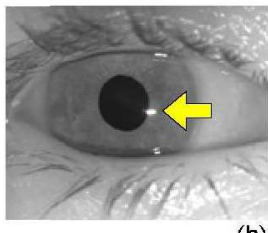

(b)

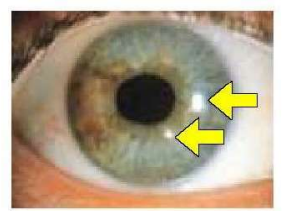

(e)

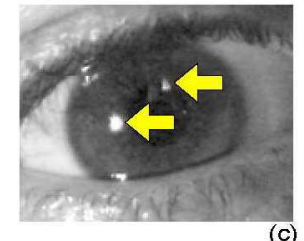

(c)

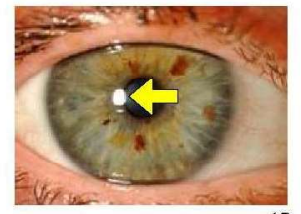

(f)
Figure 1. Reflections present in iris images acquired with different sensors and optics.

Reflections in iris images can occur in a great variety of applications and acquisition system. This phenomenon is due to the particular shape and condition of the cornea (the spherical and wet transparent surface that protects the iris and the inner eye from the outside). The light coming from windows, from screens and from the illumination system (which is required to correctly acquire the iris image) is often reflected by the cornea. Additional reflections may be caused by occlusions such as contact lenses and eye glasses.

Unfortunately, reflections tend to be superimposed on the iris patter causing difficulties in the iris acquisition. Complex iris-based biometric systems use special illumination system (single point IR illuminators, optical filters, etc.) and they require that users must be correctly positioned in front of the sensor. As such, reflections are contained in the pupil area, hence outside of the iris pattern. But, even in such cases, in low cost systems and in outdoor conditions external reflections are often present. 
The goal of this paper is to propose an innovative automated method to identify and remove reflections from the iris pattern. The proposed method can work with any close-up image of the eye, and it does not require any information concerning the iris position and segmentation.

The paper is structured as follows. Section 2 presents the state of the art, while section 3 proposes a set of features that can be used to identify the reflections in the iris image. In particular, the section shows how the radial symmetry operators can be used and how its parameters can be properly tuned with respect to the input image. Section 4 shows the application of the neural networks for the identification of the image areas containing the reflections. Then, the creation of the training dataset is discussed. Section 5 compares the results of the neural networks with respect to traditional classification paradigms (k-nearest neighbor classifiers) discussing accuracies and computational complexities.

\section{STATE OF THE ART}

The approaches present in the literature traditionally consider the reflections as the others occlusions. Typically, the methods try to evaluate the global quality of the iris acquisition by measuring a global property of the iris patter. In [4] Ma et al. anlalyze the Fourier spectra of local iris regions to characterize defocus, motion and occlusion. Zhang [5] cxamines the sharpness of the region between the pupil and the iris. Daugman [6] and Kang [7] evaluate the iris quality by estimating the energy of high spatial frequencies over the entire image region. The mentioned techniques consider only one or two global features extracted from the iris/eye image in order to estimate the quality of the image.

Differently, a local analysis of the iris has been considered in the literature to achieve a proper segmentation of occlusions. In [8] Chen et al. use the local features extracted from one iris image using a $2 \mathrm{D}$ wavelet technique. A similar local approach is present also in [4]. In [9], Zhu et al. present a technique based on the analysis of a sequence of images using wavelet coefficients.

In the literature, it is well-known that the performance of the biometric system enhances for most encoding techniques once the reflections have been compensated. Reflection compensation is typically aclieved by localizing the reflections using a segmentation technique based on a hard thresholding. The thresholding is performed using one or more features extracted from the iris pattern as described in the previous paragraph. Then, the localized regions are typically median-filtered to complete the compensation. In any case, a mask image will be created in order to mark each pixel that is supposed to belong to the iris pattern and not to occlusions (i.e., in the well known IrisCode proposed by J. Doughman [10]).

\section{FEATURE EXTRACTION}

The approach we present in this paper is based on an innovative approach for the reflection identification: a suitable set of features is extracted from the iris pattern, and an inductive classifier is then used to achieve the reflections segmentation (Figure 2). In particular we focus on a feature defined as radial symmetry map and on the usage of the neural networks as classifiers.

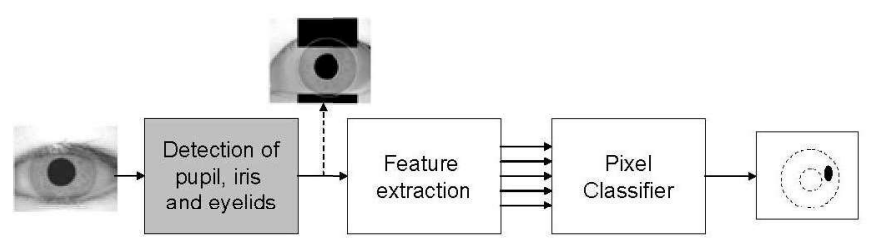

Figure 2. Structure of the proposed system for reflection identification.

The algorithm we consider to compute the radial symmetry map is the Fast Radial Symmetry Transform proposed by Loy and Zelinsky [11]. This algorithm maps the local radial symmetry of an image into a transformed image using a limited computational complexity with respect to other methods present in the literature. Due to the spherical shape of the cornea, the reflections tend to be small than the pupil and, moreover, they generally are circular-shaped. This kind of shape has a relatively high value in the radial symmetry map. Figure 3 shows the application of the map with different images.

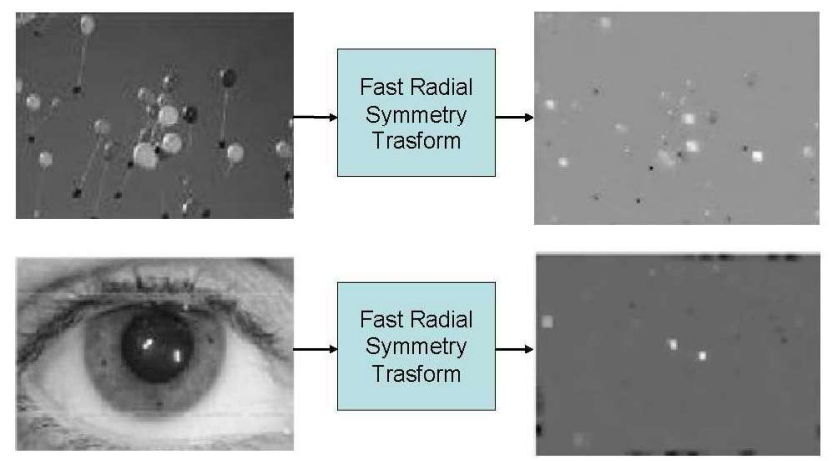

Figure 3. Application of the Fast Radial Symmetry transform to an example image (top) and to an iris image (bottom). Local maxima in the transformation image are related to objects in the input image with high radial symmetry, such as the reflections.

The radial symmetry transformation has two main parameters. The first one is an array of proposed radii $(\boldsymbol{r})$ that will be considered in the images analysis, the second one is the radial strictness parameter $(\alpha)$. In the transformed image, only symmetrical objects which have comparable radii to the ones in the $\boldsymbol{r}$ array will be enhanced (high contrast). Objects with low radial symmetry tend to be suppressed in the transformed image (low contrast). With small values of the $\alpha$ parameter (i.e., $\leq 1$ ), features present in the image, which have also bilateral symmetry, will be enhanced. With higher values of $\alpha$, only objects/features in the image with strict radial symmetry will be enhanced.

A more discriminative and significant set of features can be obtained using the radial symmetry maps, integrated with other classical ones (e.g., the normalized pixel intensity). 
This set of features can be used as input to a neural network which can perform the reflection identification better than the traditional thresholding method. The results of this technique will be given in the next section.

\section{CLASSIFICATION AND EXPERIMENTAL RESULTS}

In this section the traditional approaches are compared with the proposed technique based on the radial symmetry maps. In particular the application of neural networks has been tested with the aim of taking the advantages both of the traditional method and of the radial symmetry maps.

\section{A. The dataset and the traditional approach}

Traditionally the approaches present in the literature consider a binarization approach on the input gray level image $[8,9,12]$. This is related to the fact that the reflexes are caused by external light and, hence, they tend to be characterized by high grey intensities. This approach is very simple but it is not robust since its accuracy strongly depends on the setup of the binarization threshold. It is very difficul to fix a proper threshold suitable for different images types even using an adaptive threshold. In addition, some whit image regions may not contain any reflexes (for example th sclera region). As such, the binarization approaches tend to produce a large false detection ratio since they classify whit portions as reflections (e.g., the sclera region). In th following, we refer to this method with the name $B I N$.

In this paper we used a dataset of eye images coming fron different sensors (as described in the introduction) which ha been classified at pixel level by an expert user in two classes reflection, non-reflection. The dataset consists of $15387 \mathrm{c}$ classified pixels. The occurrence of the reflections pixels in the dataset is about $2.34 \%$. The probability of an iris pixel to be a reflection is about $6.92 \%$.

\section{B. The proposed approach}

The first implementation of the reflection identification method based on the radial symmetry maps we propose in this paper consists of a simple thresholding technique. Once the radial symmetry map has been processed, the reflections are identified by the pixel in the map which has a value higher than a fixed threshold. By using this approach, it is possible to identify the reflections from the shape information given by the radial symmetry map. In the following we refer to this method with the name RSBIN.

Since both approaches depend on the value of a threshold (which has to be suitably fixed by designers) a fair comparison can be done using the ROC curve, where the values of the false acceptance rate (FAR) and the false rejection rate (FRR) are plotted for all the possible value of the thresholds of the algorithms. By using the ROC curve it is possible to identify the best method working in the fixed ranges of FAR e FRR related to considered the application.
Figure 4 shows the ROC curves of the $B I N$ algorithm (traditional binarization) with respect to the ROC curve of the $R S B I N$ method with different $\alpha$ values. The ideal algorithm should produce FAR and FRR equal to zero for all the values of its threshold, hence its ROC curve is along the axes. The plots show very different behaviors of the two algorithms. The traditional binarization algorithm BIN provides a better behavior in the left part of the ROC curve than the proposed RSBIN. Conversely, in the right part of the ROC curve, the RSBIN provides the best results. For example, the classification error of the sclera as a reflection produced by the BIN algorithm is mapped in the ROC curve as a large FAR rate. The right plot shows the Total Classification Error of the algorithms (the sum of the FAR and FRR). The traditional binarization algorithm has a minimum of the total classification error when the threshold is set to 1 (all the image pixels are classified as non-reflections). Differently, the proposed algorithms have threshold value that can effectively minimizing the sum of the FAR and FRR. Best performance can be obtained with the method RSBIN with $\alpha=1$.
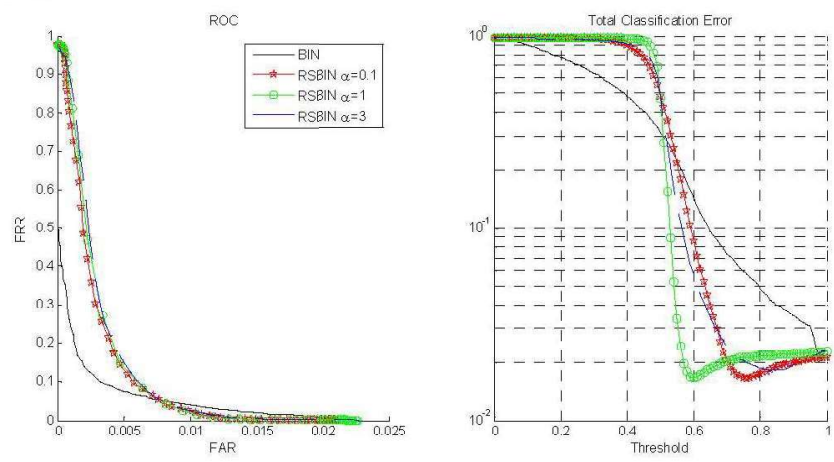

Figure 4. I eft: ROC curves of the traditional hinarization method $(B I N)$ and the proposed Radial Simmetry Binarization method (RSBIN) at different $\alpha$. Right: the Total Classification Error with respect to the threshold level.
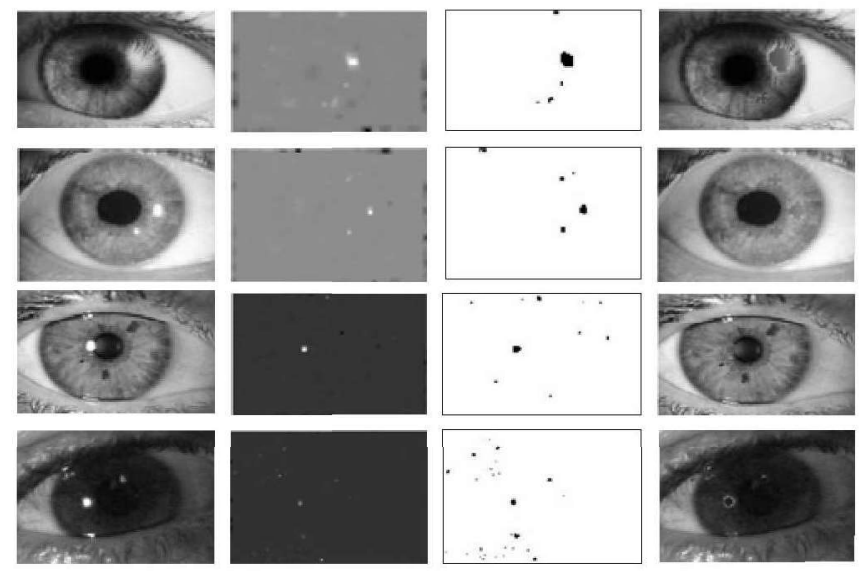

Figure 5. Application of the radial symmetry operator and reflections compensation.

Figure 4 plots for four different iris images (first column) and the corresponding radial symmetry maps (second column). Notably, this mapping properly detects the positions 
of the reflections in the iris which correspond to the positions of the local maxima. The third column plots the binarizated values of the radial symmetry maps using a fixed threshold equal to $60 \%$ of the maximum value. By selecting a higher threshold value (fixed for all images), it is possible identify with good accuracies only the reflections as shown in the last column (final compensated images). The pixcls bclonging to the reflections has been compensated by processing the mean intensity value of a 10-pixel neighborhood. This simple method is general and it can be adopted with image acquired with different resolutions, illumination type and optics.

\section{Application of the neural networks}

Computation intelligence techniques can give advantages in the reflection classification. In particular, neural networks combine in a non-linear manner the extracted features and provide the best behavior of the reflection classifier. In particular, our aim is to train a neural network to mimic the behavior or the traditional classifier in the left part of the ROC curve (Figure 4) and the behavior or the proposed classifier based on radial symmetry in the right part of the ROC curve. The basic idea is to induce the neural networks to learn the correct behavior in the different regions of the image using more than one feature in input. All tested neural network has been trained using a training dataset of 8000 classificd pixcl obtaincd by random extraction from the original dataset of 153876 pixels. The trained neural networks have been verified using the remaining partition of the dataset. In this paper it has been analyzed only neural networks working pixel-by-pixel.

The first configuration possible achieves the classification using the gray level intensity of each pixel and the corresponding value of the radial symmetry map. This input configuration has been tested using a set of feed-forward neural networks with a number of hidden neurons in the range $[1,2, \ldots, 30]$ trained ten times with the LevenbergMarquardt method and Bayesian regularisation [13],[14]. The minimum Total Error Rate has been obtained by using a feedforward neural network with 4 neurons in the hidden layer ( $F F N N 1$ in figure 6). This neural network obtained a Total Error Rate of $1.64 \%$ and its ROC curve belongs to a wide region of the error plane with FAR and FRR minor than the FAR and FRR of the $B I N$ and $R S B I N$ models respectively. A similar behavior has been obtained by a neural network with 6 neurons in the hidden layer (FFNN2 in figure 6).

A set of $\mathrm{k}$-Nearest Neighbour classifier with $\mathrm{k}$ in the $[1,3$, $5, \ldots, 15]$ range using the Euclidean norm has been tested as reference. The best results have been obtained with $k=2$. The Nearest Neighbour classifier does not have any threshold to fixed. For this reason it is not possible to process the corresponding ROC curve and only a single FAR and FRR value is available for the comparison. The best kNN tested obtained a FAR $=1.62 \%$, FRR $=1,66 \%$ and a Total Error Rate of $3.28 \%$.

The computational complexity of the $\mathrm{kNN}$ model can be estimated as about 190 times more that the best neural network by measuring ratio of the execution times of the classifiers. The execution time was measured on a real processor (IN'IEL P4 2GHz with 750MB RAM mounting the Microsoft WINDOWS Xp operating system) by removing ummecessary operating system processes. Measures were averaged over 100 presentations of the whole data set for each benchmark in order to reduce the impact of the variability of the operating system processes. The whole system has been implemented in Matlab by exploiting the available Neural Network Toolbox and PRTOOL [15].
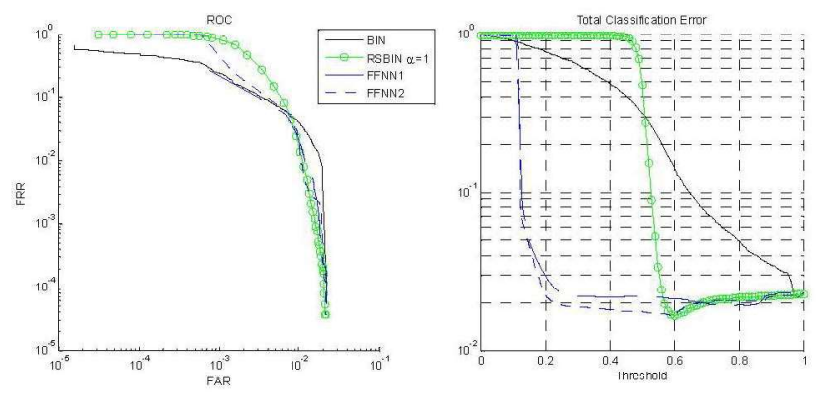

Figure 6. Application of the radial symmetry operator and reflections compensation.

\section{CONCLUSIONS}

This paper presented a methodology for reflections identification in iris biometric images. Results show that the radial symmetry map can be considered as a good feature to identify reflections. The application of this feature (using a thresholding approach) can perform a simple reflex identification. A more discriminative and significant set of features can be obtained by using the radial symmetry maps, integrated with other classical features (e.g., the normalized pixel intensity). This set of features can be used as the input of a neural network. This approach can perform the reflection identification better than traditional thresholding methods. The low computational complexity of this classifier is suitable for real time applications. The presented method is general and it can be used in any biometric system based on iris images.

\section{ACKNOWLEDGEMENT}

The author wish to thank Diego Vicoletti for his profitable contribution and discussions about the radial symmetry operator applied to the reflections identification in iris images.

\section{RFFFRFNCFS}

[1] A. K. Jain, R. Bolle, and S. Pankanti, Biometrics: Personal Identification in Networked Society. Norwell, MA: Kluwer, 1999.

[2] R. Bolle, S. Pankanti, and A. K. Jain, "Guest editorial," IEEE Computer (Special Issue on Biometrics), vol. 33, no. 2, pp. 46-49, Feb. 2000.

[3] J. Wayman, A. Jain, D. Maltoni, D. Maio, "Biometric Systems: Technology, Design and Performance Evaluation", Springer, 2005 
[4] L. Ma, T. Tan, Y. Wang, and D. Zhang, "Personal identification based on iris texture analysis," IEEE Trans. on Pattern Analysis and Machine Intelligence, vol. 25, no. 12, pp. 1519-1533, December 2003.

[5] G. Zhang and M. Salganicoff, "Method of measuring the focus of close-up image of eyes," Tech. Rep. 5953440, United States Patent, 1999.

[6] J.G. Daugman, "How iris recognition works," IEEE Trans. on Circuits and Systems for Video Technology, vol. 14, no. 1, pp. 21-30, January 2004.

[7] B.J. Kang and K.R. Park "A study on iris image restoration," Proc. Audio and Video-Based Biometric Person Authentication, vol. 3546, pp. 31-40, July 2005.

[8] Y. Chen, S. Dass, and A. Jain, "Localized iris quality using 2-d wavelets," ICBA, IIong Kong, China, 2006.

[9] X. Zhu, Y. Liu, X. Ming, and Q. Cui, "A quality evaluation method of iris images sequence based on wavelet coefficients in 'region of interest'," Proc. of the 4th Int'l Conf. on Computer and Information Technology, pp. 24-27, September 2004.
[10] J. Daugman, "Recognising Persons by Their Iris Patterns" in Advances in Biometric Person Authentication, pp. 5-25, Springer, 2004

[11] G. Loy e A. Zelinsky, Fast Radial Symmetry for Detecting Points of Interest, IEEE Transaction on Pattern Analisys and Machine Intelligence, vol. 25, no. 8, pp. 959-973, August 2003

[12] X. Liu, K. W. Bowycr, P. J. Flynn, "Experiments with an Improved Iris Segmentation Algorithm", IEEE Workshop on Automatic Identification Advanced Technologies AutoID'05, pp. 118-123, 2005

[13] F. Girosi, M. Jones, and 'I. Poggio, "Regularization theory and neural networks architectures," Neural Computation, vol. 7, no. 2, pp. 219$269,1995$.

[14] M. Hintz-Madsen, L. K. Hansen, J. Larsen, M. W. Pedersen, and M. Larsen, "Neural classifier construction using regularization, pruning and test error estimation," Neural Networks, vol. 11, no. 9, pp. 16591670,1998

[15] F. van der Heiden, R. Duin, D. de Ridder, and D. Tax, , Classification, Parameter Estimation, State Estimation: An Engineering Approach Using MatLab. NY, USA: Wiley, 2004. 